\title{
Sharp Bounds for Strains and Stresses in Uncertain Mechanical Models ${ }^{\star}$
}

\author{
Evgenia D. Popova ${ }^{1}$, Maria Datcheva ${ }^{2,3}$, Roumen Iankov ${ }^{2}$, and Tom Schanz ${ }^{3}$ \\ 1 Inst. of Mathematics \& Informatics, Bulgarian Academy of Sciences \\ 2 Institute of Mechanics, Bulgarian Academy of Sciences \\ Acad. G. Bonchev str., block $8^{1}$, block $4^{2}$, BG-1113 Sofia, Bulgaria \\ epopova@bio.bas.bg, \{iankovr, datchevam\}@yahoo.com \\ 3 Bauhaus-Universität Weimar, Germany \\ tom.schanz@bauing.uni-weimar.de
}

\begin{abstract}
Many mechanical systems, involving interval uncertainties and modelled by finite element method, can be described by parameter dependent systems of linear interval equations and response quantities depending on the system solution. A newly developed hybrid interval approach for solving parametric interval linear systems is applied to a $2 \mathrm{D}$ engineering model with interval parameters and the results are compared to other interval methods. A new technique providing very sharp bounds for the response quantities, such as element strains and stresses, is developed. The sources for overestimation when dealing with interval computations are demonstrated on a numerical plain strain example.
\end{abstract}

\section{Introduction}

All engineering design problems involve imprecision, approximation, or uncertainty to varying degrees $[1,3]$. In particular, mathematical models in environmental geomechanics cover a broad class of problems involving deformation of geomaterials. Since soil, rock and clay materials are natural ones, there is uncertainty in the material properties [6]. When the information about an uncertain parameter in form of a preference or probability function is not available or not sufficient then the interval analysis can be used most conveniently [3]. Many mechanical systems, modelled by finite element method (FEM), can be described by parameter dependent systems of linear equations. If some of the parameters are uncertain but bounded, the problem can be transformed into a parametric interval linear system which should be solved appropriately to bound the mechanical system response. This technique is usually called Interval Finite Element Method. The efforts for developing suitable interval FE methods started at mid nineties and attract considerable attention [1]. Contrary to structural mechanics, where significant effort is devoted to solving FE models involving interval uncertainties, this paper makes a first attempt to consider a 2D model with interval parameters related to soil physics and soil mechanics, applied to

\footnotetext{
* This work was supported by the NATO CLG 979541 and the Bulgarian National Science Fund under grants No. I-903/99, MM-1301/03.
} 
engineering. Since safety is an issue in environmental geomechanics, the goal is to describe the response of the system under a worst case scenario of uncertain parameters varying within prescribed bounds.

Like in FE analysis of most engineering systems [1,3], the problem considered here can be stated as follows: Given a system of linear equations, that describe the response of a discretized structural mechanical model, in the form

$$
K(p) \cdot U=F(p)
$$

and a response vector

$$
R=R(U)
$$

where $K(p)$ is the global stiffness matrix of the system, $F(p)$ is the global load vector (which might depend on uncertain parameters), $U$ is the nodal displacement vector of the total discrete model, and $p$ is the vector of uncertain input parameters which are described as intervals $p=\left\{p_{i}\right\}=\left\{\left[p_{i}^{-}, p_{i}^{+}\right]\right\}, i=1,2, \ldots, m$, where $p_{i}^{-}$denotes the lower bound and $p_{i}^{+}$denotes the upper bound for the corresponding parameter value. $R$ is the vector of response quantities which can be expressed in terms of nodal displacements, $U$, of the model. In this work we consider response quantities: element strains, $\varepsilon_{e}$, and element stresses, $\sigma_{\mathrm{e}}$,

$$
\begin{aligned}
& \varepsilon_{e}=B \cdot U_{\mathrm{e}} \quad \text { and } \\
& \sigma_{\mathrm{e}}=D\left(p_{\mathrm{e}}\right) \cdot B \cdot U_{\mathrm{e}},
\end{aligned}
$$

where $p_{\mathrm{e}}$ is the element parameter vector which is, generally, a subvector of the global parameter vector $p, U_{\mathrm{e}}$ is the subvector of element nodal displacements, amd matrices $B, D$ are defined in Sec. 3. The problem is to find the range $\left[R^{-}, R^{+}\right]$of each response quantity due to the uncertainty present in the input parameters. A key issue is how to avoid the overestimation effect caused by the dependency problem in interval analysis.

The solution of parametric linear interval equations (1) and subsequent estimation of the response parameters (3), (4) is discussed in this work. We extend the hybrid approach for solving parametric interval linear systems [2] to the subsequent bounding of the response quantities - element strains and stresses.

\section{Interval Methods}

\subsection{Solving Parametric Interval Linear Systems}

Linear algebraic systems (1) usually involve complicated dependencies between the parameters involved in the stiffness matrix $K(p)$ and the load vector $F(p)$. In this paper we assume that these dependencies are affine-linear, that is

$$
k_{i j}(p)=\lambda_{i j 0}+\sum_{\nu=1}^{m} \lambda_{i j \nu} p_{\nu}, \quad f_{i}(p)=\beta_{i 0}+\sum_{\nu=1}^{m} \beta_{i \nu} p_{\nu}
$$


where $\lambda_{i j}, \beta_{i} \in \mathbb{R}^{m+1}(i, j=1, \ldots, n)$ are numerical vectors, with $n$ being the dimension of (1). When the $m$ parameters $p_{\nu},(\nu=1, \ldots, m)$ take arbitrary values from given intervals $\left[p_{\nu}\right]$, the solution of $(1)$ is a set

$$
\Sigma^{p}=\Sigma(K(p), F(p),[p]):=\left\{U \in \mathbb{R}^{n} \mid K(p) \cdot U=F(p) \text { for some } \mathrm{p} \in[\mathrm{p}]\right\}
$$

called parametric solution set (PSS). The PSS is a subset of, and has much smaller volume than the corresponding non-parametric solution set $\Sigma^{g}=$ $\Sigma([K],[F]):=\left\{U \in \mathbb{R}^{n} \mid \exists K \in[K]=K([p]), \exists F \in[F]=F([p]): K \cdot U=F\right\}$. The simplest example of dependencies is when the matrix is symmetric. Since the solution sets have a complicated structure which is difficult to find, we look for the interval hull $\square \Sigma:=[\inf \Sigma, \sup \Sigma]$, whenever $\Sigma$ is a nonempty bounded subset of $\mathbb{R}^{n}$, or for an interval enclosure $[U]$ of $\square \Sigma$.

There are many works devoted to interval treatment of uncertain mechanical systems [1]. A main reason for some conservative results that have been obtained is that the interval linear systems (1), involving more dependencies than in a symmetric matrix, are solved by methods for nonparametric interval systems (or for symmetric matrices). Although designed quite long ago, the only available iterative method [5] for guaranteed enclosure of the PSS seems to be not known to the application scientists, or at least not applied. The parametric Rump's method is a fixed-point method applying residual iteration and accounting for all the dependencies between parameters. As it will be shown in Sec. 3, this parametric method produces very sharp bounds for narrow intervals but tends to overestimate the PSS hull with increasing the interval tolerances and the number of parameters. To overcome this deficiency, we designed a new hybrid approach for sharp PSS enclosures, that combines parametric residual iteration and exact bounds based on monotonicity properties [2].

Finding very sharp bounds (or exact in exact arithmetic) for the PSS is based on the monotonicity properties of the analytic solution $U(p)=K(p)^{-1} \cdot F(p)$. For large real-life problems, the computer aided proof of the monotonicity of $U(p)$ with respect to each parameter is based on taking partial derivatives on (1) [4]. This leads to a parametric interval linear system

$$
K(p) \frac{\partial U}{\partial p_{\nu}}=\frac{\partial F(p)}{\partial p_{\nu}}-\frac{\partial K(p)}{\partial p_{\nu}} \cdot[\tilde{U}]
$$

where $[\tilde{U}] \supseteq \Sigma^{p}$ is a PSS enclosure. Let for fixed $i, 1 \leq i \leq n$

$$
L_{-}^{u_{i}}=\left\{\nu \mid \operatorname{Sign}\left(\left[\frac{\partial U_{i}}{\partial p_{\nu}}\right]\right)=1\right\}, \quad L_{+}^{u_{i}}=\left\{\nu \mid \operatorname{Sign}\left(\left[\frac{\partial U_{i}}{\partial p_{\nu}}\right]\right)=-1\right\}
$$

and $L_{-}^{u_{i}} \cup L_{+}^{u_{i}}=\{1, \ldots, m\}$. Define numerical vectors $p^{u_{i}}, p^{-u_{i}}$ componentwise

$$
p_{j}^{u_{i}}:=\left\{\begin{array}{ll}
p_{j}^{-} & \text {if } j \in L_{-}^{u_{i}} \\
p_{j}^{+} & \text {if } j \in L_{+}^{u_{i}}
\end{array} \quad \text { and } \quad p_{j}^{-u_{i}}:=\left\{\begin{array}{ll}
p_{j}^{+} \text {if } j \in L_{-}^{u_{i}}, \\
p_{j}^{-} \text {if } j \in L_{+}^{u_{i}}
\end{array} \quad j=1, \ldots, m .\right.\right.
$$

Then the exact bounds of the PSS, $U_{i}^{-}=\inf \left\{\Sigma^{p}\right\}_{i}$ and $U_{i}^{+}=\sup \left\{\Sigma^{p}\right\}_{i}$,

$$
\left[U_{i}^{-}, U_{i}^{+}\right]=\left[K\left(p^{u_{i}}\right)^{-1} \cdot F\left(p^{u_{i}}\right), K\left(p^{-u_{i}}\right)^{-1} \cdot F\left(p^{-u_{i}}\right)\right], \quad i=1, \ldots, n
$$


can be obtained by solving at most $2 n$ point linear systems. This approach is extended in the next subsection for sharp bounding of the response quantities.

\subsection{Bounding the Response Quantities}

A naive interval approach in finding the range of the response quantities $R(U)$, such as element strains (3) and element stresses (4), under the uncertainties in input parameters is to replace the exact hull (or its enclosure) $[U]$, found by some method, into the expressions (3), (4) and to perform all interval operations. Since the nonzero components of $\left[U_{e}\right]$ depend on the uncertain parameters, the range computation of $R\left(U_{e}\right)$ implicitly involves the dependency problem implying a huge overestimation of the exact range, as will be shown in Sec. 3. To get sharp range estimations, we use the monotonicity properties of the responses. Taking partial derivatives on $(3),(4)$ we obtain

$$
\begin{aligned}
& \frac{\partial \varepsilon_{e}}{\partial p_{\nu}}=B \cdot\left[\frac{\partial U_{e}}{\partial p_{\nu}}\right] \quad(\text { since } B \text { doesn't depend on } p) \\
& \frac{\partial \sigma_{e}}{\partial p_{\nu}}=\frac{\partial D\left(p_{e}\right)}{\partial p_{\nu}} \cdot\left[\varepsilon_{e}(p)\right]+D\left(p_{e}\right) \cdot\left[\frac{\partial \varepsilon_{e}}{\partial p_{\nu}}\right] \quad \nu=1, \ldots, m,
\end{aligned}
$$

where $\left[\frac{\partial U_{e}}{\partial p_{\nu}}\right]$ is a subvector of the solution of $(5),\left[\varepsilon_{e}(p)\right]$ is the solution of $(3)$, and $\left[\frac{\partial \varepsilon_{e}}{\partial p_{\nu}}\right]$ is taken from (7). Let for fixed $i \in\left\{1, \ldots, n_{1}\right\}, j \in\left\{1, \ldots, n_{2}\right\}$, where $n_{1}, n_{2}$ are the dimensions of $\varepsilon_{e}, \sigma_{e}$ respectively.

$$
\begin{aligned}
L_{-}^{\varepsilon_{i}} & =\left\{\nu \mid \operatorname{Sign}\left(\left[\frac{\partial\left\{\varepsilon_{e}\right\}_{i}}{\partial p_{\nu}}\right]\right)=1\right\}, & L_{+}^{\varepsilon_{i}} & =\left\{\nu \mid \operatorname{Sign}\left(\left[\frac{\partial\left\{\varepsilon_{e}\right\}_{i}}{\partial p_{\nu}}\right]\right)=-1\right\} \\
L_{-}^{\sigma_{j}} & =\left\{\nu \mid \operatorname{Sign}\left(\left[\frac{\partial\left\{\sigma_{e}\right\}_{j}}{\partial p_{\nu}}\right]\right)=1\right\}, & L_{+}^{\sigma_{j}} & =\left\{\nu \mid \operatorname{Sign}\left(\left[\frac{\partial\left\{\sigma_{e}\right\}_{j}}{\partial p_{\nu}}\right]\right)=-1\right\} .
\end{aligned}
$$

Note, that the sets $L_{-}^{u}, L_{+}^{u}, L_{-}^{\varepsilon}, L_{+}^{\varepsilon}, L_{-}^{\sigma}, L_{+}^{\sigma}$ differ between themselves and for every fixed $i, j$. Define the numerical vectors $p^{\varepsilon_{i}}, p^{-\varepsilon_{i}}$, for every $1 \leqq i \leqq n_{1}$, and $p^{\sigma_{j}}, p^{-\sigma_{j}}$, for every $1 \leqq j \leqq n_{2}$, analogously to (6). Then the exact bounds of $\varepsilon_{e}$ are obtained as

$$
\left[\left\{\varepsilon_{e}\right\}_{i}^{-},\left\{\varepsilon_{e}\right\}_{i}^{+}\right]=\left[\{B\}_{i} \cdot U_{e}\left(p^{\varepsilon_{i}}\right),\{B\}_{i} \cdot U_{e}\left(p^{-\varepsilon_{i}}\right)\right], \quad i=1, \ldots, n_{1} .
$$

Note, that a rigorous very sharp enclosure can be obtained if instead of $U_{e}\left(p^{\varepsilon_{i}}\right)$, $U_{e}\left(p^{-\varepsilon_{i}}\right)$ we take the corresponding components of the rigorous interval enclosures $\left[U\left(p^{\varepsilon_{i}}\right)\right],\left[U\left(p^{-\varepsilon_{i}}\right)\right]$ of the solution to the corresponding point systems $(1)$.

For fixed $j \in\left\{1, \ldots, n_{2}\right\}$

$$
\begin{aligned}
\inf \left\{\sigma_{e}\right\}_{j} & =\left\{D\left(p_{e}^{\sigma_{j}}\right) \cdot B \cdot U_{e}\left(p^{\sigma_{j}}\right)\right\}_{j}=\left\{D\left(p_{e}^{\sigma_{j}}\right) \cdot \varepsilon\left(p_{e}^{\sigma_{j}}\right)\right\}_{j} \\
\sup \left\{\sigma_{e}\right\}_{j} & =\left\{D\left(p_{e}^{-\sigma_{j}}\right) \cdot B \cdot U_{e}\left(p^{-\sigma_{j}}\right)\right\}_{j}=\left\{D\left(p_{e}^{-\sigma_{j}}\right) \cdot \varepsilon\left(p_{e}^{-\sigma_{j}}\right)\right\}_{j} .
\end{aligned}
$$

However, the above computation of the $\sigma_{e}$ range is rigorous only in exact arithmetic. A rigorous and very sharp range enclosure of $\sigma_{e}$ in floating-point arithmetic should use rigorous interval enclosures $\left[U\left(p^{\sigma_{j}}\right)\right],\left[U\left(p^{-\sigma_{j}}\right)\right]$ of the solution to the corresponding point linear systems (1) and computations with validated interval arithmetic. 


\section{Numerical Example}

We consider an elastic material model based on the following assumptions: small strain theory is applied to describe the deformation in material; the latter is deformed elastic; material properties are isotropic; the temperature, creep and time dependent effects are not taken into account; the Elastic moduli in the material properties are considered to be uncertain and varying within prescribed bounds. For example, the prediction of soil behavior due to environmental changes, experimental procedure and measurement errors of such properties is a typical situation. Let $V$ be a representative volume and $S$ be the boundary of this volume. $S=S_{\bar{u}} \bigcup S_{\sigma}$, where $S_{\bar{u}}$ is a boundary part with prescribed displacement, and $S_{\sigma}$ is a boundary part with prescribed stresses. The governing equations are as follows:

$$
\begin{array}{lcc}
\text { Kinematics : } & \varepsilon_{i j}=\frac{1}{2}\left(u_{i, j}+u_{j, i}\right) & \text { in } V \\
\text { Equilibrium : } & \sigma_{i j, j}+b_{i}=0 & \text { in } V \\
\text { Constitutive Law : } & d \sigma_{i j}=D_{i j k l} d \varepsilon_{k l} & \text { in } V
\end{array}
$$

Boundary Cond. : $\quad u_{i}=\bar{u}_{i} \quad$ on $S_{\bar{u}}, \quad \sigma_{i j} n_{j}=\bar{T}_{i} \quad$ on $\quad S_{\sigma}$,

where $u_{i}, i=1,2,3$, are the displacements, $\varepsilon_{i j}$ is a strain tensor, $b_{i}$ is the body force, $\sigma_{i j}$ is the stress tensor, $D_{i j k l}$ is the elastic tensor, $\bar{u}_{i}$ and $\bar{T}_{i}$ are the prescribed displacements and distributed load resp., $n=\left\{n_{1}, n_{2}, n_{3}\right\}$ is the outward normal direction and $n_{i}$ are the corresponding directional cosines.

The equilibrium equation (9), relating the stress vector $\{\sigma\}$ to the body force $\{b\}$ and the boundary force specified at the boundary $S_{\sigma}$ of $V$, is formulated in terms of the unknown displacement vector $\{u\}$. Using the principle of virtual work, the general equilibrium statement can be written in a variational form $[7]$ as:

$$
\int_{V}\{\delta \varepsilon\}^{T}\{\sigma\} d V-\int_{V}\{\delta u\}^{T}\{b\} d V-\int_{S_{\sigma}}\{\delta u\}^{T}\{\bar{T}\} d S=0
$$

for a virtual displacement vector $\{\delta u\}$.

The resulting system of equations is created using mathematical formulations for FEM based on variational technique. A typical FE in the 2D plane strain case is defined by nodes $i, j, k$. Let the displacement at any point within the element $\{u\}$, be approximated by linear shape functions and nodal unknown displacement $\left\{u_{e}\right\}$. With displacements, known at all points within an element, the strains $\{\varepsilon\}$ and stresses $\sigma$ at any point of the FE can be determined by nodal unknowns. These relationships can be written in matrix notations [8] as:

$$
\begin{gathered}
\{u\}=N \cdot\left\{u_{e}\right\}, \quad\{\varepsilon\}=L \cdot N \cdot\left\{u_{e}\right\}=B \cdot\left\{u_{e}\right\}, \\
\{\sigma\}=D\left(p_{e}\right) \cdot\{\varepsilon\}=D\left(p_{e}\right) \cdot B \cdot\left\{u_{e}\right\},
\end{gathered}
$$

where $N$ is a matrix of shape functions, $L$ is a matrix of a suitable differential operators, $D\left(p_{e}\right)$ is the elastic matrix involving Elastic modulus and Poisson's 




\begin{tabular}{cc}
\hline FE & Young's modulus \\
\hline 1 & $E_{1}=200 \mathrm{GPa}$ \\
2 & $E_{2}=205 \mathrm{GPa}$ \\
3 & $E_{3}=210 \mathrm{GPa}$ \\
4 & $E_{4}=220 \mathrm{GPa}$ \\
\hline & Poisson's ratio \\
\hline 1 & $\nu_{1}=0.25$ \\
2 & $\nu_{2}=0.26$ \\
3 & $\nu_{3}=0.27$ \\
4 & $\nu_{4}=0.28$ \\
\hline
\end{tabular}

Fig. 1. 2D plane strain FEM model with 4 finite elements, where $a=1 \mathrm{~m}, q=$ $1000 \mathrm{KN} / \mathrm{m}^{2}$, and each $\mathrm{FE}$ has a different material property

ratio, $p_{e}$ denotes a parameter (or parameter vector), related to the material properties, to be considered as uncertain in the subsequent analysis.

Substituting Eqs.(11) and (12) into eq. (10) and taking into account that eq. (10) is valid for any virtual displacement, for an element $e$, we obtain a system of parametric linear equations

$$
K^{e}\left(p_{e}\right) \cdot u_{e}=F^{e}
$$

where $K^{e}\left(p_{e}\right)=\int_{V} B^{\top} D\left(p_{e}\right) B d V$ is the element stiffness matrix which is function of the uncertain material properties, $F^{e}=\int_{V} N^{\top}\{b\} d V+\int_{S} N^{\top}\{\bar{T}\} d S$ is the element load vector. It could also depend of some uncertain parameters. The final parametric global stiffness matrix and global load vector based on FEM matrices (13) is obtained by using an element-by-element technique [8].

We consider a 2D plain strain example presented on Fig. 1. The model is discretized with four triangular FEs and the kinematic boundary conditions are presented on the figure. The corresponding material and geometrical data are also given therein. It is assumed that in each FE the Young's modulus, $E_{i}$, is an independent uncertain parameter varying within an interval.

Three different parametric interval linear systems (1) are considered that involve: a) four uncertain parameters - Young's modulus for each FE of the model is uncertain and varies independently in an interval $\left[p_{i}\right]=\left[E_{i}-\Delta, E_{i}+\Delta\right]$, $i=1, \ldots, 4$; b) two uncertain parameters $-E_{1}=E_{2}$ varying in $\left[p_{1}\right]=\left[E_{1}-\right.$ $\left.\Delta, E_{1}+\Delta\right]$ and $E_{3}=E_{4}$ varying in $\left[p_{2}\right]=\left[E_{3}-\Delta, E_{3}+\Delta\right]$; c) one parameter assuming that the four FEs of the model have same Elastic modulus, $E$, varying in an interval $[p]=[E-\Delta, E+\Delta]$, where $\Delta$ is the degree of uncertainty measured in $\%$ from the corresponding nominal value. The three parametric systems are solved for different values of the tolerance $\Delta=10 \%, 5 \%, 1 \%$, and $0.1 \%$. 
Table 1. Percentage by which Rump's method overestimates the exact hull of the PSS to (1) with varying number of parameters and varying parameter tolerances

\begin{tabular}{|c|c|c|c|c|c|c|c|c|c|c|c|c|}
\hline \multirow[b]{3}{*}{$\Delta$} & \multicolumn{3}{|c|}{$6^{t h} \mathrm{DOF}$} & \multicolumn{3}{|c|}{$8^{t h} \mathrm{DOF}$} & \multicolumn{3}{|c|}{$10^{t h} \mathrm{DOF}$} & \multicolumn{3}{|c|}{$12^{t h} \mathrm{DOF}$} \\
\hline & \multicolumn{3}{|c|}{ parameters: } & \multicolumn{3}{|c|}{ parameters: } & \multicolumn{3}{|c|}{ parameters: } & \multicolumn{3}{|c|}{ parameters: } \\
\hline & 4 & 2 & 1 & 4 & 2 & 1 & 4 & 2 & 1 & 4 & 2 & 1 \\
\hline $10 \%$ & 15.87 & 14.54 & .16 & 19.45 & 14.50 & 9.16 & 20.01 & 19.20 & 9.16 & 27.65 & $19.19 \mathrm{~s}$ & .16 \\
\hline $5 \%$ & 7.93 & 7.36 & .79 & 9.88 & 7.36 & 4.79 & 10.14 & 9.81 & 4.79 & 14.38 & 9.81 & .79 \\
\hline $1 \%$ & 1.60 & 1.50 & .99 & 2.02 & 1.50 & 0.99 & 2.07 & 2.01 & 0.99 & 2.99 & 2.01 & .99 \\
\hline $.1 \%$ & 0.16 & 0.15 & .10 & 0.20 & 0.15 & 0.10 & 0.21 & 0.20 & 0.10 & 0.30 & 0.20 & .10 \\
\hline
\end{tabular}

Table 2. For the model with 4 uncertain parameters, the percentage by which a straightforward interval evaluation of (3) for the 4th FE based on: (R) Rump's enclosure; (M) exact hull of $U(p)$, overestimates the exact range of the element strains. $(\mathrm{M} / \mathrm{R})$ presents the ratio in $\%$ between the two strain estimations

\begin{tabular}{ccc}
\hline$\Delta$ & 2 & 3 \\
$10 \%$ & \multicolumn{2}{c}{ component } \\
\hline $\mathrm{R}$ & 99.40 & 98.15 \\
$\mathrm{M}$ & 99.21 & 97.56 \\
$\mathrm{M} / \mathrm{R}$ & 24.97 & 24.02 \\
\hline
\end{tabular}

\begin{tabular}{ccc}
\hline$\Delta$ & 2 & 3 \\
$5 \%$ & \multicolumn{2}{c}{ component } \\
\hline $\mathrm{R}$ & 99.30 & 97.75 \\
$\mathrm{M}$ & 99.20 & 97.44 \\
$\mathrm{M} / \mathrm{R}$ & 12.85 & 12.31 \\
\hline
\end{tabular}

\begin{tabular}{ccc}
\hline$\Delta$ & 2 & 3 \\
$1 \%$ & \multicolumn{2}{c}{ component } \\
\hline $\mathrm{R}$ & 99.22 & 97.41 \\
$\mathrm{M}$ & 99.19 & 97.34 \\
$\mathrm{M} / \mathrm{R}$ & 2.65 & 2.53 \\
\hline
\end{tabular}

\begin{tabular}{ccc}
\hline$\Delta$ & 2 & 3 \\
$.1 \%$ & \multicolumn{2}{c}{ component } \\
\hline $\mathrm{R}$ & 99.20 & 97.32 \\
$\mathrm{M}$ & 99.19 & 97.32 \\
$\mathrm{M} / \mathrm{R}$ & 0.27 & 0.25 \\
\hline
\end{tabular}

A Mathematica package IntervalComputations 'LinearSystems ' [2], providing a variety of functions for solving parametric and nonparametric interval linear systems in validated interval arithmetic, and supporting the hybrid interval approach, is used for the computations. Due to the boundary conditions, only the 6th, 8th, 10th, and 12th degree of freedom (DOF) of $U(p)$ are nonzero. We compare the results, obtained by the parametric iteration method, to the exact interval hull of the displacements. The overestimation of Rump's enclosure is measured in $\%$ as $100\left(1-\omega\left(\square \Sigma^{p}\right) / \omega([U])\right)$, where $\square \Sigma^{p}$ is the exact hull of the corresponding parametric solution set, $[U]$ is the interval enclosure, obtained by the parametric iteration method, and $\omega(\cdot)$ is the width of an interval, defined as $\omega\left(\left[a_{-}, a^{+}\right]\right)=a^{+}-a^{-}$. The percentage by which the iterative solution enclosure overestimates the exact hull of the corresponding parametric solution set is presented in Table 1. The computationally efficient iterative procedure gives sharp enclosures for small number of parameters and thin intervals for the uncertainties. However, the overestimation grows with increasing the number of parameters or the width of the intervals.

For the model with 4 uncertain parameters, a straightforward interval evaluation of (3) and (4) was done basing on interval estimations for the element displacements. Two interval estimations for the element displacements were used: interval enclosure, obtained by the parametric iteration method, and the exact interval hull. The results for the element strains are presented in Table 2. The results for the first component of the strains are not given since the component 
is zero. A huge overestimation of the strains range is demonstrated, which is due to the parameter dependencies between the components of $U_{e}$. The dependency, accumulated in the interval enclosure $\left[U_{e}\right]$ cannot be taken into account by the interval computation $B \cdot\left[U_{e}\right]$. It is remarkable, that the difference in the interval estimations of $U$ (by Rump's method and the exact hull) is obscured by the inherited dependencies between the displacement components.

The naive range estimation of the element stressess showed similar results. Note that the expression (4) involves additional dependencies due to the parameters in the matrix $D\left(p_{e}\right)$. Accounting for the dependencies in $D\left(p_{e}\right)$ reduces the overestimation by $30-50 \%$.

Since the considered model is small, the exact interval hulls and the exact ranges were computed by symbolic-algebraic methods in the environment of Mathematica. Our hybrid approach produced very sharp bounds for both the solution set of the displacements system and the ranges of the response parameters. The difference to the corresponding exact values was within $10^{-16}-10^{-18}$.

\section{Conclusions}

The interval analysis methods, presented in this paper, can be applied to all engineering problems whose behavior is governed by parametric interval linear systems and response quantities of type (1), (2). The hybrid interval approach, proposed in Sec. 2, is based on numerical proof of monotonicity properties and on a fast iteration method for enclosing PSS. By using validated interval computations, this approach has the additional property that, owing to an automatic error control mechanism, every computed result is guaranteed to be correct.

The Mathematica package [2] should be extended by suitable functions automating the computations of the response quantities. Further research toward improving the computational efficiency of the method would allow large industrial applications.

\section{References}

1. Muhanna R.; Mullen R.: Short Review for the Latest Development of Interval

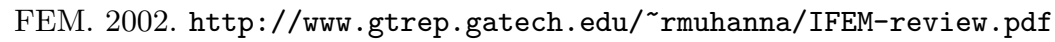

2. Popova, E.: Parametric Interval Linear Solver. Numerical Algorithms, submitted.

3. Rao S. S.; Berke L.: Analisys of Uncerttain Structural Systems Using Interval Analysis. AIAA Journal, 35 (1997) 4, 727-735.

4. Rohn, J.: Interval Linear Equations with Dependent Coefficients. Reliable Computing mailing list, 2002. http://www.ms.mff.cuni.cz/ ${ }^{\sim}$ rohn/letter/letter.ps

5. Rump, S.: Verification Methods for Dense and Sparse Systems of Equations. In: Herzberger, J.(ed.): Topics in Validated Computations. Elsevier Science B. V. (1994) 63-135.

6. Schanz T.: Zur Modellierung des mechanischen Verhaltens von Reibungsmaterialien. Habilitationsschrift, Institut fuer Geotechnik, Stuttgart, 1998.

7. Washizu K.: Variational Methods in Elasticity and Plasticity. Pergamon Press, New York, 1982.

8. Zienkiewicz O. C.: The Finite Element Method in Engineering Science. McGrawHill, London, 1971. 